\title{
Cutaneous Herpetic Infection in an Immuno-Compromised Individual- An Unusual Presentation
}

\author{
Dr. Mousumi Paul ${ }^{1 *}$, Dr. Tapan Majumdar ${ }^{2}$, Dr. Jaharlal Baidya ${ }^{3}$
}

${ }^{1}$ Medical Officer-MD Microbiology, Tripura Health Service, Agartala Government Medical College \& GBP Hospital, Agartala, Pin no. 799006, Tripura, India

${ }^{2}$ Professor and Head, Department of Microbiology, Agartala Government Medical College \& GBP Hospital, Agartala, Pin no. 799006, Tripura India ${ }^{3}$ Professor, Department of Obstetrics and Gynecology, Agartala Government Medical College \& GBP Hospital, Agartala, Pin no. 799006, Tripura India

*Corresponding author: Dr. Mousumi Paul

Abstract

Herpes simplex virus (HSV) has various presentations, depending on the patient's immune status, age and the route of transmission. In adults, HSV-1 is found generally in the oral area and HSV-2 is commonly found in genital area. HSV2 without genital lesion is uncommon. Patients with immunodeficiency are at an increased risk of developing severe herpes simplex virus (HSV) infection and atypical presentation. Herein we report a unique case of cutaneous herpetic infection during postpartum period of a diabetic women caused by HSV-2 without genital involvement.

Keywords: Herpes simplex virus, immunodeficiency, atypical presentation, postpartum period.

Copyright @ 2020: This is an open-access article distributed under the terms of the Creative Commons Attribution license which permits unrestricted use, distribution, and reproduction in any medium for non-commercial use (NonCommercial, or CC-BY-NC) provided the original author and source are credited.

\section{INTRODUCTION}

Herpes simplex virus 1 and 2 are doublestranded DNA viruses which belong to the alphaherpesvirus subfamily of Herpesviridae [1]. HSV1 and HSV-2 are neurotropic herpesviruses that cause vesicular mucocutaneous eruptions. They both establish latency in peripheral ganglia and can reactivate to cause episodic outbreaks [2-4]. HSV-1 more commonly causes infections around the mouth while HSV-2 more commonly causes genital infections. They are transmitted by direct contact with body fluids or lesions of an infected individual. Transmission may still occur when symptoms are not present. Genital herpes is classified as a sexually transmitted infection. It may be spread to an infant during childbirth. After infection, the viruses are transported along sensory nerves to the nerve cell bodies, where they reside lifelong. Causes of recurrence may include: decreased immune function, stress, and sunlight exposure. Oral and genital herpes is usually diagnosed based on the presenting symptoms. The diagnosis may be confirmed by viral culture or detecting herpes DNA in fluid from blisters. Testing the blood for antibodies against the virus can confirm a previous infection but will be negative in new infections [5].

\section{Case Report}

A 30 years old female delivered a healthy girl child by cesarean section at obstetrics \& gynecology department of our hospital. She was complaining of multiple bullae formation on left leg on her second postpartum day along with mild fever.

Present history- She was a known case of uncontrolled diabetes for last 15 years for which she was under insulin therapy for last few months during her pregnancy period. Bullae were multiple in numbers and scattered over her left leg \& lower part of thigh only (figure 1). It was non-itchy \& mild painful along with low grade of fever. No such history of similar illness in past (no h/o chicken pox/ herpes), no h/o drug allergy was present. There was no herpetic skin manifestation or any other symptoms in the newborn girl child.

On examination- She was afebrile, vitals were stable. On local examination- multiple bullae were present over left leg \& thigh. The characteristic of fluid was clear, thin serous type \& yellowish in color. Base of the lesion was red in color and tender on palpation. No mucosal lesion or no lesion over genitalia was found on examination. She was started treatment with injection ceftriaxone 1gram twice daily. After that a physical and dermatologist call was initiated and differential diagnosis were given- 
1. Drug allergy/ adverse drug reaction

2. Toxin necrotizing epidermolysis

3. Sepsis

4. Deep Vein Thrombosis

After that injection ceftriaxone was changes to injection Meropenem ( 8 hourly) by physician. But with this also there was no improvement.

Microbiological examination- After that a microbiologist calls was initiated. After detailed examination, the fluid content of the lesion was first aspirated and gram staining was done and material was inoculated at blood agar and macConkey agar for bacteriological culture isolation. Then deroofing of those bullae was performed and scrapping materials were collected from the base of lesions. Giemsa staining and gram staining was performed with this materials.

2 set of blood from 2 separate venipuncture sites were collected under all aseptic conditions for blood culture. Serum for HSV-1, HSV-2, VZV IgM detection were also performed.

\section{Lab investigation}

TLC- 13,800/cumm, DLC- N- $86 \%$, L- $12 \%$, M-0 \% , E- $1 \%$, B- $0 \%, \mathrm{Hb}-9.8$ gm $\%$

CRP- Positive $200 \mathrm{mg} / \mathrm{dl}$

HSV-1 IgM- Negative, HSV-2 IgM-positive, VZV IgM- Negative

FBS-145 mg/dl, PPBS- 185 mg/dl, HbA1c-9.4

\section{Microbiological examination findings}

Aspirated fluid- Macroscopically - Yellowish, clear, thin serous type of fluid. Gram staining finding of aspirated fluid- Pus cell- 4-5 /HPF, No organism was seen. Bacteriological culture -No growth of organism after 48 hours of incubation. Blood culture (BecT ALERT) - No growth of organism after 7 days of incubation.

Scrapping of base of the bullae: smear (Tzanck smear) prepared \& Giemsa staining was doneMultinucleated giant cell with intranuclear inclusion body found (figure-2, 3).

From the clinical history, clinical findings and microbiological investigations diagnosis was made as a case of "Herpetic bullae most probably caused by Herpes Simplex Virus-2"

Following our diagnosis, on that same day treatment was started with Acyclovir infusion @ $5 \mathrm{mg} /$ day, 8 hourly with local application of Acyclovir ointment. With this treatment she improved and was discharged from hospital after 7 days of treatment (figure-4).

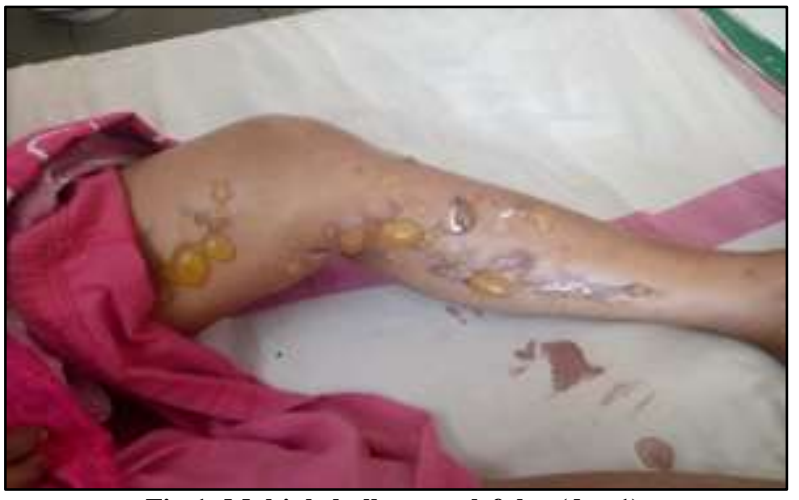

Fig-1: Multiple bullae over left leg (day 1)

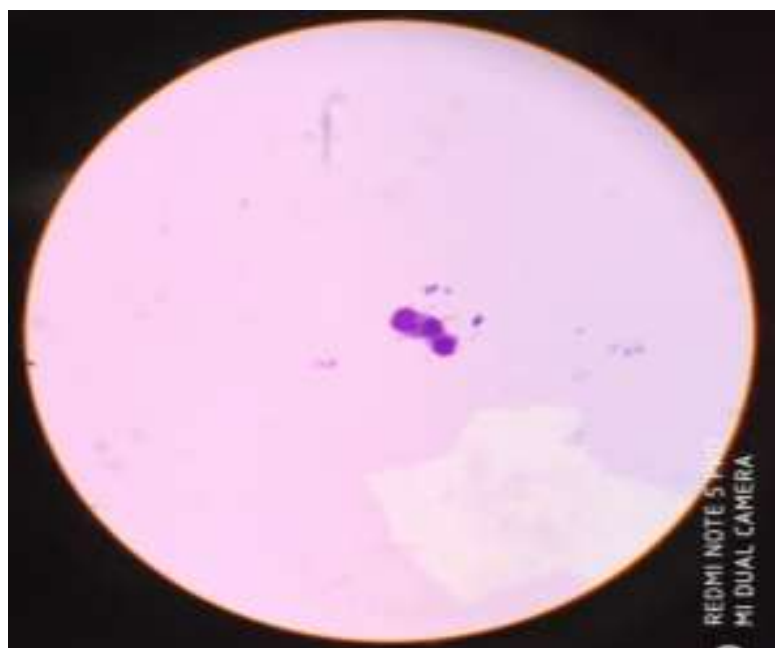

Fig-2: Intranuclear inclusion body seen in giemsa staining of scrapping from base of the bullae (X1000)

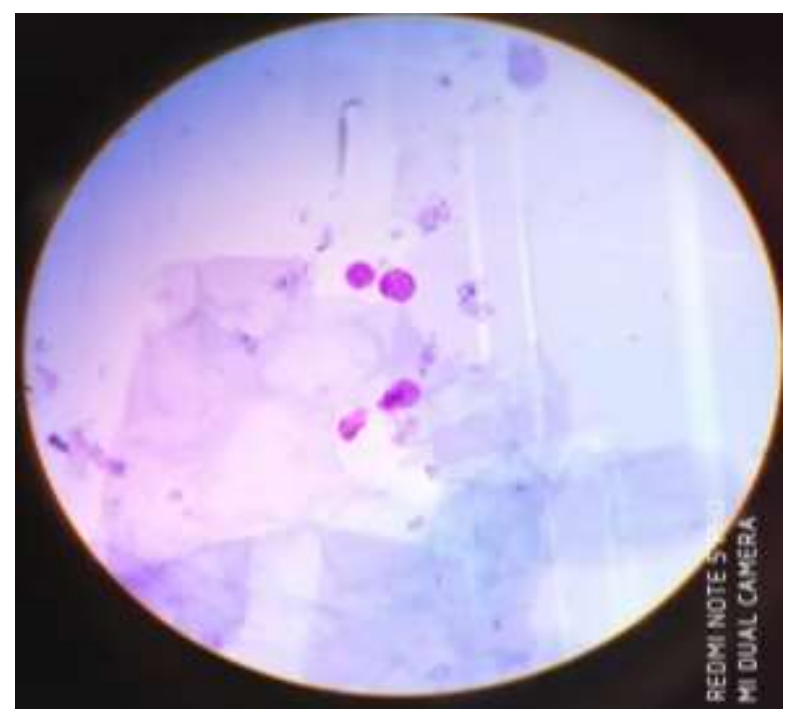

Fig-3: Multinucleated squamous epithelial cell (Tzanck cell) seen in giemsa staining of scrapping from base of the bullae (X1000) 


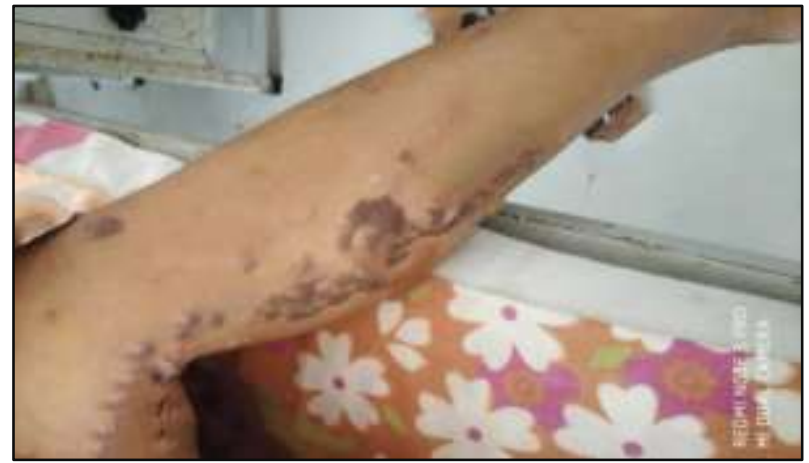

Fig-4: Scar developed after 5 days of antiviral treatment

\section{DISCUSSION}

The incubation period for herpes infection is 2 to 12 days (average is 4 days) after exposure. Herpes simplex virus is an important cause of neonatal infection, which can lead to death or long-term disabilities. Rarely in the uterus, transmission occurs frequently during the delivery. The greatest risk of transmission to the fetus and the newborn occurs in case of an initial maternal infection contracted in the second half of pregnancy. The risk of transmission of maternalfetal-neonatal herpes simplex can be decreased by performing a treatment with antiviral drugs or resorting to a caesarean section in some specific cases. The purpose of this study is to provide recommendations on management of herpes simplex infections in pregnancy and strategies to prevent transmission from mother to fetus and also to consider the atypical presentation of cutaneous manifestations of HSV-2 in immunecompromised individual.

\section{REFERENCE}

1. De Vivo C, Bansal MG, Olarte M, Grossman ME. Concurrent herpes simplex type 1 and varicellazoster in the V2 dermatome in an immunocompetent patient. Cutis. 2001;68(2):1202.

2. Kinchington PR, Leger AJ, Guedon JM, Hendricks RL. Herpes simplex virus and varicella zoster virus, the house guests who never leave. Herpesviridae. 2012;3(1):5. doi: 10.1186/20424280-3-5.

3. Meier JL, Straus SE. Comparative biology of latent varicella-zoster virus and herpes simplex virus infections. The Journal of infectious diseases. 1992;166 Suppl 1(Suppl 1):S13-23.

4. McDonald PJ, Krishnan-Natesan S. Concurrent Reactivation of VZV and HSV-2 in a Patient with Uncontrolled Diabetes Mellitus: A Case Report. European Journal of Medical Case Reports. 2017;1(3):xx-Xx. https://doi.org/10.24911/ejmcr/1/26

5. "Genital herpes- CDC fact sheet" cdc.gov. December 8, 2014. Archived from the original on 31 December 2014. Retrieved 31 December 2014. 